\title{
The impact of genetic risk for Alzheimer's disease on the structural brain networks of young adults
}

\author{
Anastasia Mirza-Davies ${ }^{1,2}$, Sonya Foley ${ }^{2}$, Xavier Caseras ${ }^{3}$, Emily Baker, ${ }^{4}$ Peter \\ Holmans, ${ }^{3}$ Valentina Escott-Price, ${ }^{4}$ Derek K. Jones ${ }^{2}$, Judith R. Harrison ${ }^{2,5}$ and Eirini \\ Messaritaki $i^{2,6}$ \\ 1. School of Medicine, University Hospital Wales, Heath Park, Cardiff University, Cardiff, CF14 4XW, UK \\ 2. Cardiff University Brain Research Imaging Centre (CUBRIC), School of Psychology, Cardiff University, Maindy \\ Road, Cardiff, CF24 4HQ, UK \\ 3. MRC Centre for Neuropsychiatric Genetics and Genomics, Division of Psychological Medicine and Clinical \\ Neurosciences, Cardiff University, UK \\ 4. Dementia Research Institute \& the MRC Centre for Neuropsychiatric Genetics and Genomics, Cardiff University, \\ CF24 4HQ, UK. \\ 5. Institute for Translational and Clinical Research, Newcastle University, Newcastle-Upon-Tyne, NE1 7RU, UK \\ 6. BRAIN Biomedical Research Unit, School of Medicine, Cardiff University, Maindy Road, Cardiff CF24 4HQ, UK
}

\begin{abstract}
To facilitate pre-symptomatic diagnosis of late-onset Alzheimer's disease, non-invasive imaging biomarkers could be combined with genetic risk information. In this work, we investigated the structural brain networks of young adults in relation to polygenic risk for Alzheimer's disease, using magnetic resonance imaging (MRI) and genotype data for 562 19year-old participants from the Avon Longitudinal Study of Parents and Children. Diffusion MRI data were acquired on a 3T scanner, and the data were used to perform whole-brain tractography. The resulting tractograms were used to generate structural brain networks, using the number of streamlines and the diffusion tensor fractional anisotropy as edge weights. This was done for the whole-brain connectome, and for the default mode, limbic and visual subnetworks. The mean clustering coefficient, mean betweenness centrality, characteristic path length, global efficiency and mean nodal strength were calculated for these networks, for each participant. The hubs of the networks were also identified, and the connectivity of the rich-club, feeder and local connections was also calculated. Polygenic risk scores (PRS), estimating the burden of genetic risk carried by an individual, were calculated both at genome-wide level and for nine specific disease pathways. The correlation coefficients were calculated between the PRS and a) the graph theoretical metrics of the structural networks and b) the rich-club, feeder and local connectivity of the whole-brain networks.
\end{abstract}

In the visual subnetwork, the mean nodal strength was negatively correlated with the genomewide PRS including the APOE locus $\left(r=-0.19, p=1.3 \times 10^{-5}\right)$, the mean betweenness centrality was positively correlated with the pathway-specific PRS for plasma lipoprotein particle assembly including the APOE locus $\left(r=0.16, p=9.2 \times 10^{-4}\right)$, and the mean clustering coefficient was negatively correlated with the tau protein binding PRS including the APOE locus $\left(r=0.16, p=9.2 \times 10^{-4}\right)$. In the default mode network, the mean nodal strength was negatively correlated with the genome-wide PRS, including the APOE locus $(r=-0.14$, $\left.p=1.5 \times 10^{-3}\right)$. The rich-club and feeder connectivities were negatively correlated with the genome-wide PRS including the APOE locus $\left(r=-0.16, p=3.7 \times 10^{-4} ; r=-0.15, p 8.8 \times 10^{-4}\right.$, respectively). Our results indicate small changes in the brain connectome of young adults at risk of developing Alzheimer's disease in later life. 
Keywords: ALSPAC, Alzheimer's disease, polygenic risk score, brain structure, brain networks, diffusion MRI, tractography

\section{Introduction}

Alzheimer's disease (AD) is a progressive neurodegenerative disorder that affects over 35 million people world-wide (Prince et al., 2013). It leads to severe cognitive impairment and the inability of patients to function independently. There is a pressing need to identify non-invasive biomarkers that could facilitate pre-symptomatic diagnosis when disease-modifying therapies become available. Although a minority of early-onset AD cases are caused by mutations in specific genes with autosomal dominant inheritance (Tanzi, 2012), the majority of AD has a complex genetic architecture and is highly heritable (Gatz et al., 2006), with different genes conveying different amounts of risk. Genome-wide Association Studies (GWAS) have implicated many Single Nucleotide Polymorphisms (SNPs) (Kunkle et al., 2019), of which the apolipoprotein e4 allele (APOE4) confers the greatest risk (Lambert et al., 2013; Yu et al., 2014; Farrer et al., 1997), but is neither necessary nor sufficient to cause AD (Sims et al, 2020). AD GWAS have also found evidence that specific biological processes, or disease pathways, such as cell trafficking, beta amyloid production, tau protein regulation and cholesterol transport are involved (Kunkle et al., 2019; Jones et al., 2010). Polygenic risk scores (PRS), which aggregate risk loci genome-wide (Wray et al., 2014), are highly predictive of AD (Sleegers et al., 2015; Xiao et al., 2015; Yokohama et al., 2015; Escott-Price et al., 2015; Escott-Price et al., 2017; Tosto et al., 2017; Chaudhury et al., 2018; Cruchaga et al., 2018; Harrison et al., 2020; Altmann et al., 2020) and have been widely used in the search for biomarkers for the disease (Harrison et al., 2020).

Obtaining reliable biomarkers in a non-invasive manner is very valuable because it can be better tolerated by participants compared to more invasive methods (Prestia et al., 2013; Zhang et al., 2012). Magnetic resonance imaging (MRI) can non-invasively measure characteristics of the brain's structure. Diffusion-weighted MRI (dMRI, Le Bihan et al., 2006) has allowed mapping of the brain's white-matter (WM) tracts, enabling the study of the human brain as a network of cortical and subcortical areas connected via those tracts. Via these techniques, alterations in the brain of $A D$ patients and of people at risk of developing $A D$ have been identified. AD patients exhibit axonal loss in tracts associated with certain default mode network (DMN) nodes (Mito et al., 2018). They also exhibit increased characteristic path length and decreased intramodular connections in functional and structural brain networks compared to healthy controls (Dai et al., 2018). The DMN is altered in the presence of AD pathology (Dai et al., 2018) where a decrease in its connectivity has been observed (Mohan et al., 2016; Badhwar et al., 2017). The diffusion tensor fractional anisotropy in the cingulum and of the splenium of the corpus callosum is reduced in AD patients compared to controls (Zhang et al., 2007). Structural covariance brain networks, in which the edges are calculated as the correlations between the node volumes, show decreased small-worldness in AD (John et al, 2017). Increased shortest path length and clustering coefficient, as well as decreased global and local efficiency have been observed in the structural brain networks of AD patients (He et al., 2008; Lo et al., 2010). These results suggest that, in addition to the AD pathology preferentially affecting specific brain areas, $A D$ is a disconnection syndrome.

Cognitively healthy middle-aged and older carriers of AD risk (genetic or otherwise) also exhibit alterations in brain structure. Decreased hippocampal volume and cortical thickness have been associated with high AD PRS (Mormino et al., 2016; Corlier et al., 2017; Li et al., 2017). Ageing APOE4 carriers have reduced local structural connectivity at the precuneus, medial orbitofrontal cortex and lateral parietal cortex (Brown et al., 2011). APOE4 status also affects the clustering coefficient and the local efficiency of structural brain networks (Ma et al., 2017). Middle-aged adults with genetic, family and lifestyle risks of developing AD have a hub in their structural connectome that is not present in the structural connectome of people with 
no such risks of developing AD (Clarke et al., 2020). Significant functional connectivity differences in the brain networks implicated in cognition were seen in middle-aged individuals with a genetic risk for AD (Goveas et al., 2013). The DMN also exhibits changes in mature (Fleisher et al., 2009) and young APOE4 carriers (Filippini et al., 2009). A PRS composed of immune risk SNPs is associated with a thinner regional cortex in healthy older adults at risk of developing AD (Corlier et al., 2017). Other studies have also investigated the effect of $A D$ PRS on brain structure (Lupton et al., 2016; Hayes et al., 2017; Harrison et al., 2016; Sabuncu et al., 2012), finding alterations associated with increased genetic burden. Some of the studies have also used disease pathways to inform the PRS (Caspers et al., 2020; Ahmad et al., 2018). A few studies have also identified alterations in the brain of young AD-risk carriers. The hippocampal volume and the fractional anisotropy of the right cingulum are altered in young adults with increased risk of developing AD (Foley et al. 2017), and their precuneal volume is reduced ( $\mathrm{Li}$ et al., 2018). Increased functional connectivity and hippocampal activation in a memory task was observed in the DMN of young, cognitively normal APOE4 carriers (Filippini et al., 2009). Young APOE4 carriers also showed increased activation (measured via fMRI) in the medial temporal lobe compared to non-carriers, while performing a memory task (Dennis et al., 2010).

Despite the evidence that a) there are alterations in the brain networks of AD patients, and $b$ ) there are functional and structural changes in the brains of young adults at risk of developing $A D$, the structural brain networks of young adults at risk of $A D$ have not been studied. Our work fills that gap, by investigating structural brain networks of young adults at different risks of developing $A D$, where the risk is evaluated both via GWAS and via specific risk pathways. We hypothesise that the localized alterations in the structure of the brain of young adults at risk of $A D$ would present themselves as changes in their structural brain networks. We investigate the network corresponding to the whole-brain connectome, as well as the DMN, the limbic and visual subnetworks, because those subnetworks are known to be affected in AD (Power et al., 2011; Deng et al. 2016; Hansson et al., 2017; Badhwar et al., 2017; Wang et al. 2019). We also investigate the hubs of the whole-brain connectome and their interconnectivity.

\subsection{Hypotheses}

We hypothesise that increased risk of $A D$ would lead to increased characteristic path length for those networks, and an increased mean clustering coefficient, in agreement with the alterations these measures present in AD. We also hypothesize that the interconnectivity of the hubs would be reduced for increased risk of AD. Given the young age of the participants, we expect any observed alterations to be small. Any identified changes could be followed up in a longitudinal study of the same cohort, and possibly lead to important biomarkers that indicate disease onset or progression, or inform early preventative interventions in adults at risk of $A D$.

\section{Materials and Methods}

\subsection{Participants}

The Avon Longitudinal Study of Parents and Children (ALSPAC) is a pregnancy and birth cohort established to identify the factors influencing child health and developmental outcomes. Pregnant women resident in Avon, UK with expected dates of delivery 1st April 1991 to 31st December 1992 were invited to take part in the study. The initial number of pregnancies enrolled is 14,541 (for these at least one questionnaire has been returned or a "Children in Focus" clinic had been attended by 19/07/99). Of these initial pregnancies, there was a total of 14,676 foetuses, resulting in 14,062 live births and 13,988 children who were alive at 1 year of age. 
Between the ages of 18 to 24 years, a subset of ALSPAC offspring were invited to participate in three different neuroimaging studies; the ALSPAC Testosterone study (Liao et al., 2021; Patel et al., 2020; $n=513$, mean age at attendance 19.62 years, range 18.00 to 21.50 years), the ALSPAC Psychotic Experiences (PE) study (Fonville et al., 2015; Drakesmith et al., 2015; Drakesmith et al., 2016; Drakesmith et al., 2019; $n=252$, mean age at attendance 20.03 years, range 19.08 to 21.52 years), and the ALSPAC Schizophrenia Recall-by-Genotype (SCZ-RbG) study (Lancaster et al., 2019; $n=196$, mean age at attendance 22.75 years, range 21.12 to 24.55 years). Scanning protocols were harmonised across sub-studies where possible, and all data were acquired at Cardiff University Brain Research Imaging Centre (CUBRIC).

We analysed data from 562 individuals (mean age 19.81 years, SD 0.02 years; $62 \%$ male) from those ALSPAC neuroimaging studies (Boyd et al., 2013; Fraser et al., 2013; Sharp et al., 2020). Please note that the study website contains details of all the data that is available through a fully searchable data dictionary and variable search tool (http://www.bristol.ac.uk/alspac/researchers/our-data). Written informed consent was collected for all participants in line with the Declaration of Helsinki. Ethical approval for the neuroimaging studies was received from the ALSPAC Ethics and Law Committee and the local NHS Research Ethics Committees. Informed consent for the use of data collected via questionnaires and clinics was obtained from participants following the recommendations of the ALSPAC Ethics and Law Committee at the time.

\subsection{MRI acquisition}

MRI data were acquired using a GE HDx 3T system (GE Healthcare, Milwaukee W1) at CUBRIC. Axial T1-weighted images were acquired using a 3D fast spoiled gradient recalled sequence $\left(\mathrm{TR}=8 \mathrm{~ms}, \mathrm{TE}=3 \mathrm{~ms}, \mathrm{TI}=450 \mathrm{~ms}\right.$, flip angle $=20^{\circ}$, matrix size $\left.=256 \times 192 \times 159\right)$ to aid co-registration. Diffusion-weighted images were acquired with a twice refocused spinecho echo-planar imaging sequence parallel to the anterior-posterior commissure and the acquisition was peripherally gated to the cardiac cycle. Data were collected from 60 slices of $2.4 \mathrm{~mm}$ thickness (FOV=230 mm, matrix size $96 \times 96, T E=87 \mathrm{~ms}$, b-values 0 and 1200 $\mathrm{s} / \mathrm{mm}^{2}$ ) using parallel imaging (ASSET factor $=2$ ) encoding along 30 isotopically distributed directions according to vectors taken from the International Consortium for Brain Mapping protocol (Jones et al., 1999). For 219 of those participants, the diffusion-weighted images were acquired using 60 directions. For those participants, a subsample of the optimal 30 directions were used, alongside the first three images with b-value equal to 0 (see Foley et al., 2018, for further details; Afzali et al., 2021; Jones et al., 1999).

\subsection{Data processing and tractography}

Data pre-processing was performed as described by Foley et al. (2018). To summarise, T1 structural data were down-sampled to $1.5 \times 1.5 \times 1.5 \mathrm{~mm}^{3}$ resolution. Eddy-current and participant motion correction were performed with an affine registration to the non-diffusionweighted images (Leemans and Jones, 2009). Echo-planar imaging of the diffusion-weighted data was performed, warping the data to the down-sampled T1-weighted images (Irfanoglu et al., 2012). RESTORE (Chang et al., 2005), RESDORE (Parker et al., 2013a) and free water correction (Pasternak et al., 2009) algorithms were run. Whole-brain tractography was performed for each data set using the damped Richardson-Lucy pipeline (Dell'Acqua et al., 2010) and in-house MATLAB code (Parker et al., 2013b). The criteria used for termination of the tracts were: angle threshold of $>45^{\circ}$, fibre orientation density function peak $<0.05$ and fractional anisotropy $<0.2$.

\subsection{Network construction}


We used the Automated Anatomical Labelling (AAL) (Tzourio-Mazoyer et al., 2002) to define the 90 cortical and subcortical areas of the cerebrum that correspond to the nodes of the structural networks. The WM tracts linking those areas are the connections, or edges, of the networks. The network generation was performed in ExploreDTI-4.8.6 (Leemans et al., 2009). We generated two connectivity matrices for each participant, one in which the edges are weighted by the number of streamlines (NS) and one in which they are weighted by the mean fractional anisotropy of the diffusion tensor along the streamlines (FA). Both these metrics have been shown to result in measures of connectivity that exhibit heritability (Arnatkeviciute et al., 2020), repeatability (Yuan et al., 2018; Roine et al., 2019; Messaritaki et al., 2019; Dimitriadis et al., 2021) and functional relevance (Honey et al., 2009; Goni et al., 2014; Messaritaki et al., 2021). To reduce the possible number of false connections, structural connections reconstructed with 5 or fewer streamlines were discarded from the analysis. Furthermore, to avoid our results being dependent on this choice of threshold, the analysis was repeated for this threshold being from 1 to 12 streamlines. A graphical representation of this part of the analysis is shown in Fig. 1.

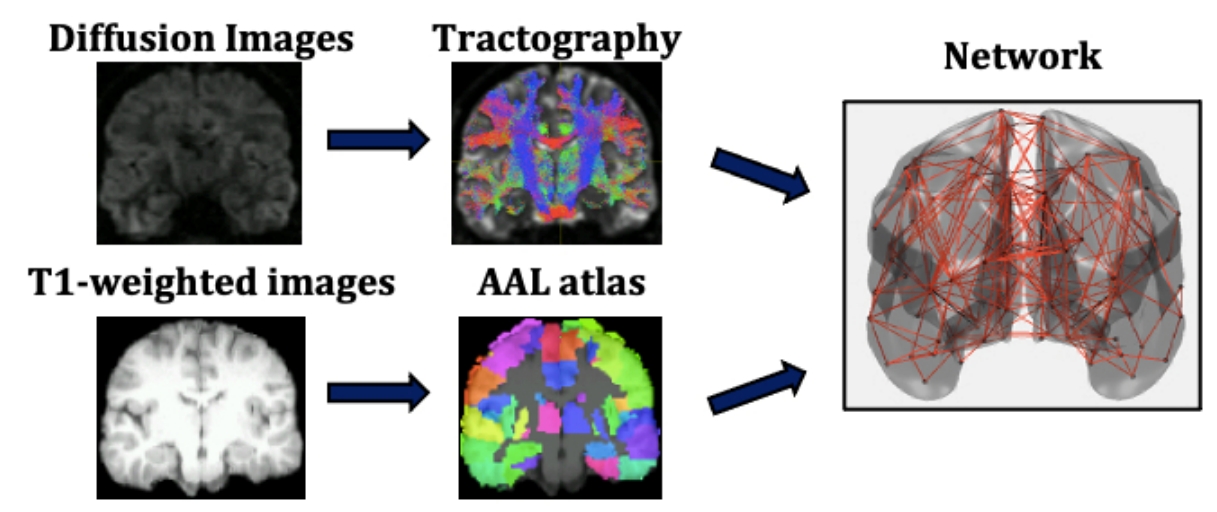

Figure 1: Analysis that leads from the MR images to the structural brain networks. This analysis is repeated for each participant individually.

In addition to the whole-brain connectome, we derived the DMN, the limbic subnetwork and the visual subnetwork, by selecting the edges that connect only the nodes in those subnetworks. The AAL atlas regions for the subnetworks are listed in Table 1 (Power et al., 2011).

\begin{tabular}{|ll|l|l|}
\hline & \multicolumn{1}{|c|}{ DMN } & \multicolumn{1}{c|}{ VIMBIC } & \multicolumn{1}{c|}{ VISUAL } \\
\hline Middle Orbitofrontal Gyr & Middle Frontal Gyr & Hippocampus & Inferior Temporal Gyr \\
Thalamus & Inferior Orbitofrontal Gyr & Amygdala & Fusiform Gyr \\
Precuneus & Superior Frontal Gyr & Anterior Cingulate Gyr & Lingual Gyr \\
Superior Orbitofrontal Gyr & Inferior Frontal Gyr (Triangular) & Middle Cingulate Gyr & Calcarine Fissure \\
Anterior Cingulate Gyr & Inferior Frontal Gyr (Opercula) & Posterior Cingulate Gyr & Cuneus \\
Middle Cingulate Gyr & Middle Occipital Gyr & Parahippocampal Gyr & Middle Occipital Gyr \\
Posterior Cingulate Gyr & Angular Gyr & Olfactory & Superior Occipital Gyr \\
Hippocampus & Parahippocampal Gyr & Insula & Inferior Occipital Gyr \\
\hline
\end{tabular}

Table 1: Nodes of the AAL atlas included in the DMN, limbic and visual subnetworks. The nodes from both the left and right hemispheres are included.

\subsection{Graph theory and network analysis}

The Brain Connectivity Toolbox (BCT, Rubinov and Sporns, 2010) was used to calculate graph theoretical metrics for the structural brain networks of all participants. A detailed 
description of graph theoretical metrics is provided by Rubinov and Sporns, 2010, but we provide here a brief explanation of the ones we use, for completeness.

The clustering coefficient of a node is equal to the number of existing edges among the neighbours of the node divided by the number of all possible edges and is a measure of how interconnected the node's neighbours are. The degree of a node is the number of edges that stem from that node. The betweenness centrality of a node is the number of shortest paths (connecting pairs of nodes) that the node belongs to in the network. The nodal strength is the sum of the weights of the edges stemming from a node. These four graph theoretical metrics are node-specific. To derive network-wide measures, their mean values over all the nodes in the network are used. The characteristic path length of a network is the mean value of the steps along the shortest paths that connect all possible pairs of nodes in the network. The global efficiency of the network is proportional to the sum of the inverse shortest path lengths over all pairs of nodes in the network and is related to how efficiently the nodes of the network can exchange information. In contrast to the previous measures mentioned, the characteristic path length and the global efficiency are network-wide, rather than node-specific, measures. Finally, the local efficiency of a node is calculated the same way as the global efficiency of the subnetwork that consists of the node's neighbours.

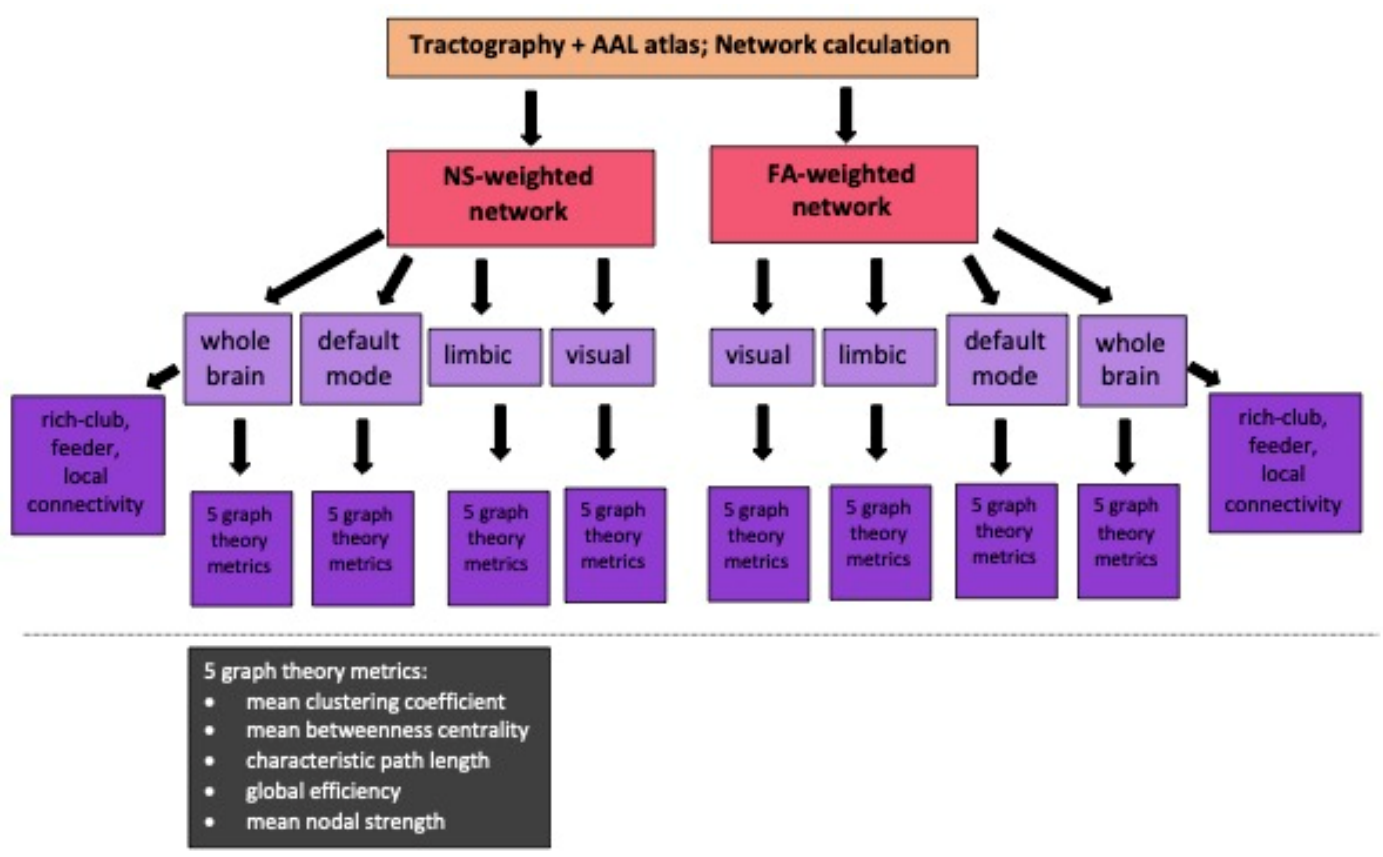

Figure 2: Diagram showing the sub/networks used in our analysis and the graph theoretical and connectivity metrics that are correlated with the PRS. NS = number of streamlines, FA = fractional anisotropy of the diffusion tensor.

For our analysis, we calculated the mean clustering coefficient, mean betweenness centrality, characteristic path length, global efficiency and mean nodal strength. The expectation is that, if changes to the topological organisation are a result of increased risk of developing $A D$, then the mean clustering coefficient, global efficiency and mean nodal strength will decrease, and the characteristic path length will increase, for increased risk. In order to remove metrics that represent redundant information from our analysis, we calculated the Pearson correlation between all pairs of graph theoretical metrics for each network and excluded from further analysis metrics that exhibited correlation coefficients of 0.85 or higher. 
In order to investigate the hubs of the networks, we also calculated the local efficiency and the degree of each node. This allowed us to calculate the hub-score, or hubness, of each node for the whole-brain network. Instead of using a single measure for identifying hubs (for example only the node degree or only the betweenness centrality as is sometimes done), we used a composite measure as proposed by Betzel et al. (2014). Specifically, we normalized the node degree, nodal strength, betweenness centrality and local efficiency for each participant and averaged the normalized values for each node. That average was the hubness of the node.

The hubness of each node was averaged over all participants, to derive the mean node hubness. Hub nodes were defined as those with mean node hubness greater or equal to the average of the mean node hubnesses plus one standard deviation, according to van den Heuvel and Sporns (2011). The hub nodes comprise a rich club of nodes. The rich-club connectivity was calculated for each participant by summing the strength of the edges that connect the hub nodes only. The feeder connections, i.e., the connections that link one hub node and one non-hub node, were also identified. The feeder connectivity was also calculated for each participant, as the sum of the strength of the feeder connections. Finally, the local connections were identified as the connections that link non-hub nodes only. The local connectivity was the sum of the strength of the local connections. We stress that the rich-club, feeder and local connectivities are defined for the whole-brain network.

\subsection{Polygenic risk score calculation}

Genome data were provided by the University of Bristol. ALSPAC participants were genotyped using the Illumina HumanHap550 quad genome-wide SNP genotyping platform by 23andMe subcontracting the Wellcome Trust Sanger Institute (WTSI, Cambridge, UK) and the Laboratory Corporation of America (Burlington, North Carolina, USA). Participants were excluded from analysis if they had minimal or excessive heterozygosity, genotyping completeness $<97 \%$, or if they were of non-European ethnicity. Quality control parameters were as follows: Minor allele frequency (MAF) $>0.01$; Individual call rate $>95 \%$, Hardy Weinberg Equilibrium (HWE) $\left(\mathrm{P}>5 \times 10^{-7}\right)$. Polygenic risk scores were calculated according to the International Schizophrenia Consortium method (Purcell et al., 2009). Training data were taken from the latest genetic meta-analysis of Alzheimer's disease (Kunkle et al, 2019) comprising of 94,437 cases and controls. In our sample, SNPs with low MAF $<0.1$ and imputation quality $<0.9$ were removed. Data were then pruned for SNPs in linkage disequilibrium (LD) using genetic data analysis tool PLINK (Chang et al., 2015) using the clumping function (--clump). This aimed to remove SNPs in LD within a 500 kilobase window, retaining only the most significantly associated SNPs. Scores were generated in PLINK using the -score command.

To compute pathway-specific PRS, nine pathway groups were taken from Kunkle et al. (2019), who matched lists of SNPs to genes and tested them for enrichment within gene functional categories. The pathway groups were as follows: protein-lipid complex assembly, regulation of beta-amyloid formation, protein-lipid complex, regulation of amyloid precursor protein catabolic process, tau protein binding, reverse cholesterol transport, protein-lipid complex subunit organisation, plasma lipoprotein particle assembly and activation of the immune response. The lists of SNPs were matched to SNPs in our target dataset. Then the data was clumped and scored as described above.

A previous study found that an AD PRS computed with p-value threshold $\left(\mathrm{P}_{\mathrm{T}}\right)$ of 0.001 explained the most variance in structural (non-network) neuroimaging phenotypes of healthy young adults (Foley et al. 2017). Therefore, our primary analysis used $\mathrm{P}_{\mathrm{T}}=0.001$ to select relevant SNPs from the discovery sample. For our secondary analysis, 7 different progressive training $\mathrm{P}_{\mathrm{T}} \mathrm{S}$ were computed $(0.00001 ; 0.0001 ; 0.01 ; 0.05 ; 0.1 ; 0.3 ;$ and 0.5$)$. Lower $\mathrm{P}_{\mathrm{T}}$ indicates that SNPs are more significantly associated with AD case status in the training dataset. Two versions of each score were calculated, including and excluding the $A P O E$ 
locus. This was done to assess the effect of PRS without APOE and the effect of $A P O E$ within the PRS.

Through this method, we ended up with 20 different PRS: genome-wide with and without $A P O E$, and each of the nine pathway-specific PRS with and without $A P O E$. Each of these PRS further corresponds to 8 values for the $\mathrm{P}_{\mathrm{T}} \mathrm{S}$, as described above.

\subsection{Statistical analyses}

Correlations between graph theoretical metrics and the genome-wide PRS (APOE included) and the nine pathway-specific PRS (APOE included) were calculated in MATLAB (MATLAB and Statistics Toolbox Release, 2015b and 2021a; The MathWorks, Inc, Massachusetts, United States). Correlations were also calculated between the rich-club, feeder and local connectivity versus the 10 PRS scores. The participant gender and the diffusion scan type (30 vs 60 diffusion gradient directions) were controlled for in calculating the correlations. Data points that had Cook's distance higher than 3 times the mean Cook's distance (Cook, 1977) were removed from the calculation. Our primary analysis used $\mathrm{P}_{\mathrm{T}}=0.001$. We also looked at the rest of the $\mathrm{P}_{\mathrm{T}}$ thresholds, as is standard practice (de Leeuw et al., 2015). Resulting $p$ values were corrected for multiple comparisons using false-discovery-rate (FDR) correction (Benjamini and Yekutieli, 2005). The correction was applied over the 5 graph theoretical metrics of all four networks, the rich-club, feeder and local connectivities, and the 10 PRS (i.e., the genome-wide plus the 9 pathway-specific ones) for each $\mathrm{P}_{\mathrm{T}}$. If a significant association was found between a PRS and the graph theoretical metrics or connectivities, correlations were also calculated with the PRS excluding the APOE locus, to assess whether the correlations were purely due to that locus. To exclude the possibility that our results are confounded by population stratification, we repeated our analyses using the first ten principal components derived from common alleles as covariates.

\section{Results}

\subsection{Networks}

The whole-brain, default mode, limbic and visual subnetworks for one participant are shown in Fig. 3 (NS-weighted networks) and Fig. 4 (FA-weighted networks). The relative strength of the connections depends on the edge-weighting and has an impact on the graph theoretical metrics of the networks. Given the differences observed between NS- and FA-weighted networks, performing the analysis for both these edge-weightings is warranted. 

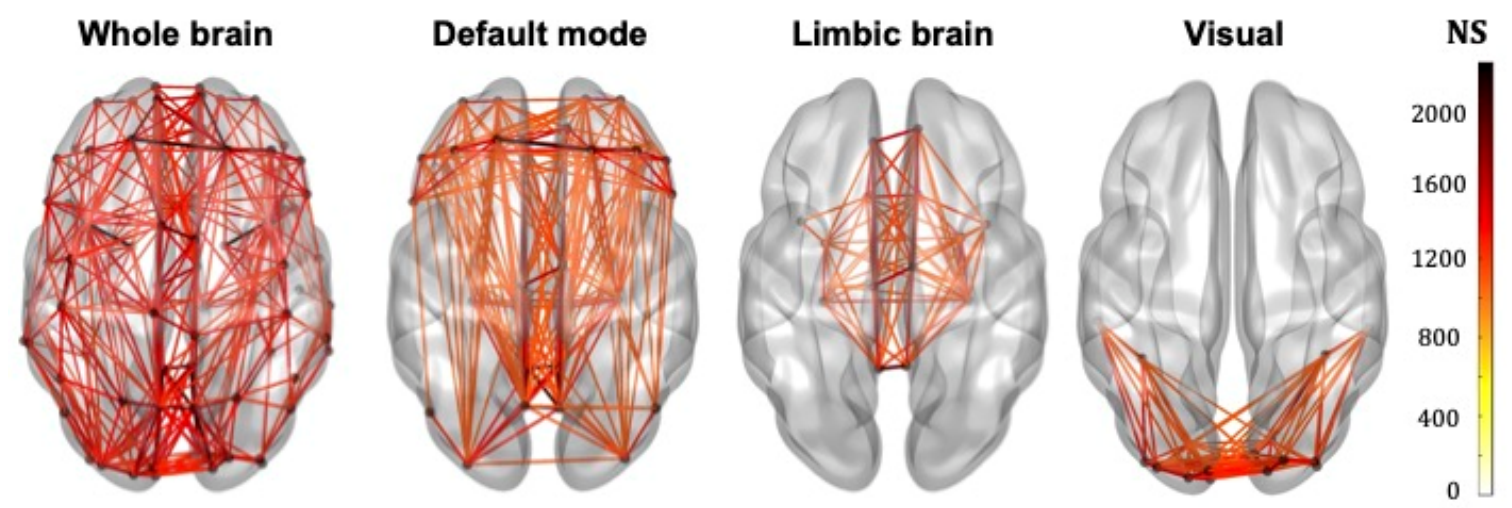

Figure 3: Whole-brain, DMN, limbic and visual subnetworks, for NS-weighted networks, from the data of one participant. The lines represent the edges (connections) between brain areas.
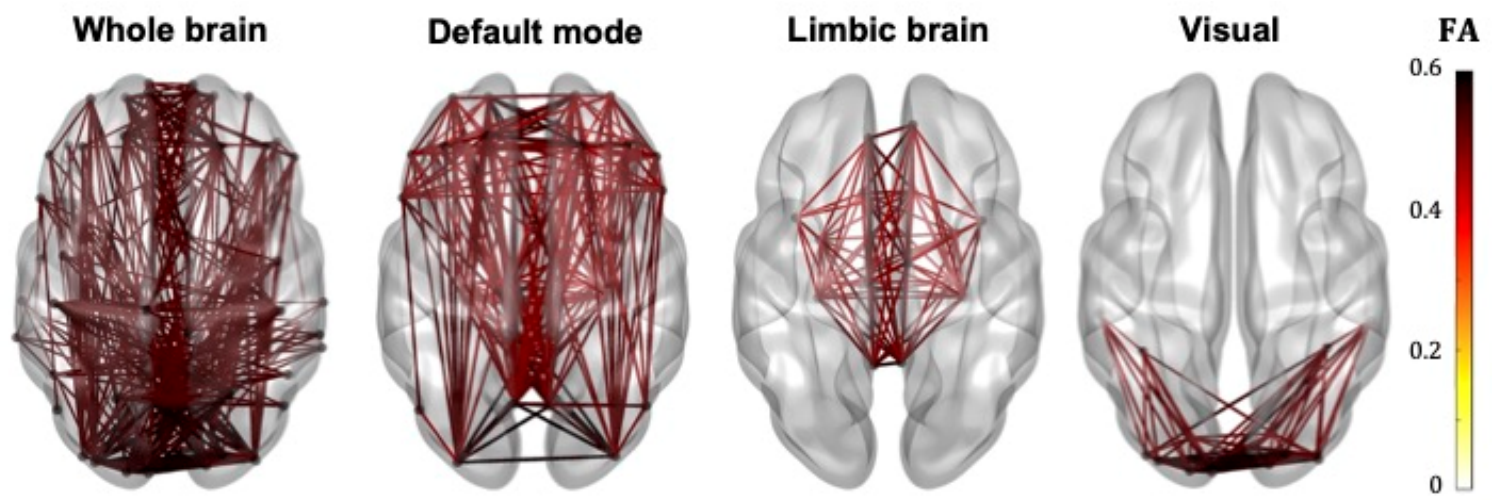

Figure 4: Whole-brain, DMN, limbic and visual subnetworks, for FA-weighted networks, from the data of one participant. The lines represent the edges (connections) between brain areas.

The correlation coefficients between graph theoretical metrics of the NS-weighted whole-brain network were high between the characteristic path length and the global efficiency $(r=-0.97, p$ $\left.<10^{-10}\right)$, between the characteristic path length and the mean nodal strength $\left(r=-0.93, p<10^{-}\right.$ $\left.{ }^{10}\right)$, and between the global efficiency and the mean nodal strength $\left(r=0.97, p<10^{-10}\right)$. The characteristic path length and the global efficiency of the NS-weighted whole-brain network were, therefore, excluded from further analysis.

The correlation coefficient between the mean nodal strength and the global efficiency of the NS-weighted default-mode network was high $\left(r=0.95, p<10^{-10}\right)$. The global efficiency of the NS-weighted default-mode network was, therefore, excluded from further analysis.

The correlation coefficient between the mean nodal strength and the global efficiency of the NS-weighted limbic subnetwork was high $\left(r=0.95, p<10^{-10}\right)$. The global efficiency of the NSweighted limbic subnetwork was, therefore, excluded from further analysis.

The correlation coefficients between graph theoretical metrics of the NS-weighted visual subnetwork were high between the characteristic path length and the global efficiency $(r=-$ $\left.0.91, p<10^{-10}\right)$, between the characteristic path length and the mean nodal strength $(r=-0.87$, $\left.p<10^{-10}\right)$, and between the global efficiency and the mean nodal strength $\left(r=0.98, p<10^{-10}\right)$. The characteristic path length and the global efficiency of the NS-weighted visual subnetwork were, therefore, excluded from further analysis. 
The correlation coefficients between graph theoretical metrics of the FA-weighted whole-brain network were high between the characteristic path length and the global efficiency $(r=-0.99, p$ $\left.<10^{-10}\right)$, between the characteristic path length and the mean nodal strength $\left(r=-0.91, p<10^{-}\right.$ $\left.{ }^{10}\right)$, and between the global efficiency and the mean nodal strength $\left(r=0.91, p<10^{-10}\right)$. The characteristic path length and the global efficiency of the FA-weighted whole-brain network were, therefore, excluded from further analysis.

The correlation coefficients between graph theoretical metrics of the FA-weighted defaultmode network were high between the characteristic path length and the global efficiency $(r=-$ $\left.0.98, p<10^{-10}\right)$, between the characteristic path length and the mean nodal strength $(r=-0.92$, $\left.p<10^{-10}\right)$, and between the global efficiency and the mean nodal strength $\left(r=0.93, p<10^{-10}\right)$. The characteristic path length and the global efficiency of the FA-weighted default-mode network were, therefore, excluded from further analysis.

The correlation coefficients between graph theoretical metrics of the FA-weighted limbic network were high between the characteristic path length and the global efficiency $(r=-0.98, p$ $\left.<10^{-10}\right)$, between the characteristic path length and the mean nodal strength $\left(r=-0.95, p<10^{-}\right.$ $\left.{ }^{10}\right)$, between the global efficiency and the mean nodal strength $\left(r=0.94, p<10^{-10}\right)$, and between the mean betweenness centrality and mean nodal strength $\left(r=-0.92, p<10^{-10}\right)$. The characteristic path length, the global efficiency and the mean nodal strength of the FAweighted limbic network were, therefore, excluded from further analysis.

The correlation coefficients between graph theoretical metrics of the FA-weighted visual subnetwork were high between the characteristic path length and the global efficiency $(r=-$ $\left.0.98, p<10^{-10}\right)$, between the characteristic path length and the mean nodal strength $(r=-0.96$, $\left.p<10^{-10}\right)$, and between the global efficiency and the mean nodal strength $\left(r=0.96, p<10^{-10}\right)$. The characteristic path length and the global efficiency of the FA-weighted visual subnetwork were, therefore, excluded from further analysis.

Table 2 summarises the metrics used in the subsequent analyses.

\begin{tabular}{|c|l|l|}
\hline & \multicolumn{1}{|c|}{ NS-weighted } & \multicolumn{1}{c|}{ FA-weighted } \\
\hline Whole-brain & $\begin{array}{l}\text { mean clustering coefficient } \\
\text { mean betweenness centrality } \\
\text { mean nodal strength } \\
\text { rich-club connectivity } \\
\text { feeder connectivity } \\
\text { local connectivity }\end{array}$ & $\begin{array}{l}\text { mean clustering coefficient } \\
\text { mean betweenness centrality } \\
\text { mean nodal strength } \\
\text { rich-club connectivity } \\
\text { feeder connectivity } \\
\text { local connectivity }\end{array}$ \\
\hline Default-mode & $\begin{array}{l}\text { mean clustering coefficient } \\
\text { mean betweenness centrality } \\
\text { mean nodal strength } \\
\text { characteristic path length }\end{array}$ & $\begin{array}{l}\text { mean clustering coefficient } \\
\text { mean betweenness centrality } \\
\text { mean nodal strength }\end{array}$ \\
\hline Limbic & $\begin{array}{l}\text { mean clustering coefficient } \\
\text { mean betweenness centrality } \\
\text { mean nodal strength } \\
\text { characteristic path length }\end{array}$ & $\begin{array}{l}\text { mean clustering coefficient } \\
\text { mean betweenness centrality }\end{array}$ \\
\hline Visual & $\begin{array}{l}\text { mean clustering coefficient } \\
\text { mean betweenness centrality } \\
\text { mean nodal strength }\end{array}$ & $\begin{array}{l}\text { mean clustering coefficient } \\
\text { mean betweenness centrality } \\
\text { mean nodal strength }\end{array}$ \\
\hline
\end{tabular}

Table 2: Metrics used in the analysis for each network.

\subsection{Whole-brain connectome}


No statistically significant correlations between graph theoretical metrics of the whole-brain network and the PRSs were found to survive multiple comparison correction.

\subsection{Default-mode network}

For our primary analysis $\left(\mathrm{P}_{\mathrm{T}}=0.001\right)$, no statistically significant correlations between the PRS and the graph theoretical metrics of the DMN survived multiple-comparison correction. The following correlations, however, did survive multiple comparison correction:

For $\mathrm{P}_{\mathrm{T}}=0.3$, the mean nodal strength of the NS-weighted DMN was correlated with the genome-wide PRS, including APOE, $\left(r=-0.14, p=1.5 \times 10^{-3}\right)$. When the APOE locus was excluded from the analysis, the correlation persisted $\left(r=-0.14, p=1.6 \times 10^{-3}\right)$. The correlations also persisted when the analysis was repeated for NS thresholds between 1 and 12 .

For $\mathrm{P}_{\mathrm{T}}=0.01$, the mean betweenness centrality of the FA-weighted DMN was correlated with the activation of the immune response PRS, including APOE, $\left(r=-0.16, p=1.2 \times 10^{-4}\right)$. When the APOE locus was excluded from the analysis, the correlation persisted $\left(r=-0.15, p=4.5 \times 10^{-}\right.$ $\left.{ }^{4}\right)$. The correlations also persisted when the analysis was repeated for NS thresholds between 1 and 12.

Repeating the analyses for the DMN using the first ten principal components derived from common alleles as covariates did not change these results.

All these results are shown in Fig. 5.
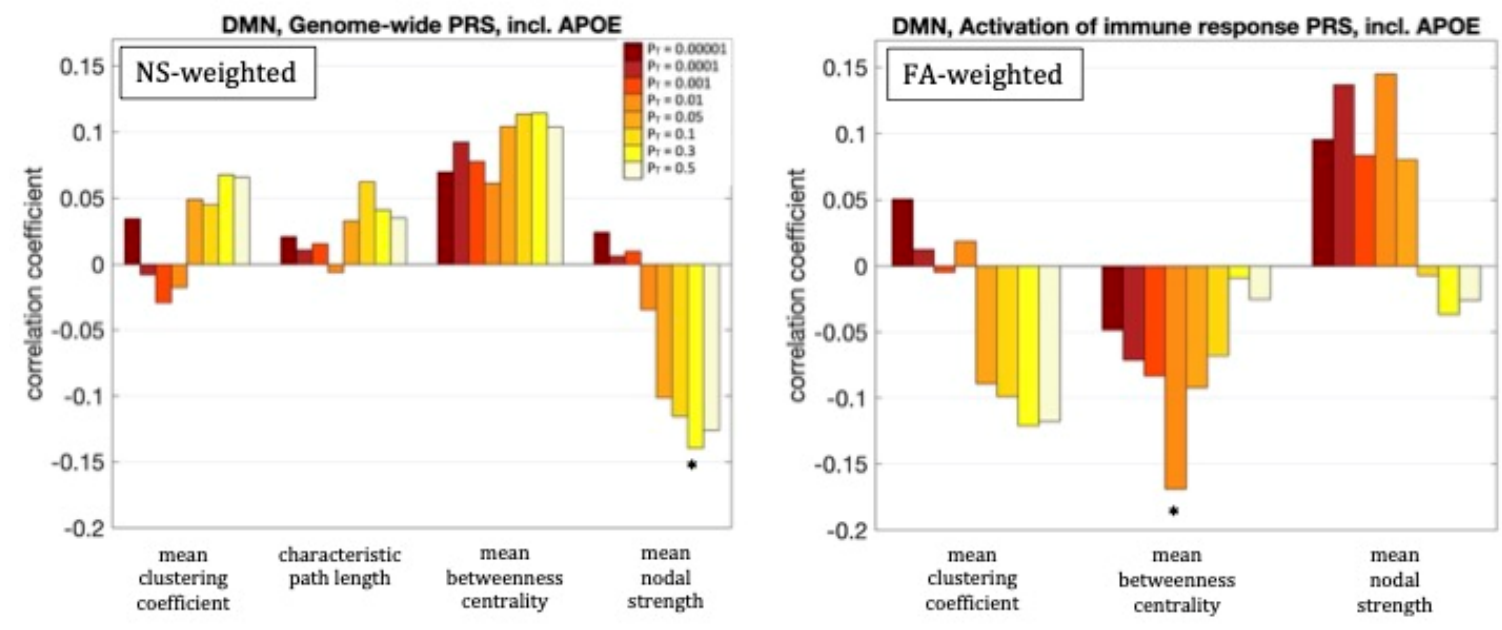

Figure 5: Correlation coefficients between the graph theoretical metrics of the default-mode network and the genome-wide PRS including APOE for the 8 different values of $\mathrm{P}_{\mathrm{T}}$. The asterisk indicates the instances in which the $p$-value survived multiple comparison correction.

\subsection{Limbic subnetwork}

No statistically significant correlations between graph theoretical metrics of the limbic subnetwork and the PRSs were found to survive multiple comparison correction.

\subsection{Visual subnetwork}


For our primary analysis $\left(\mathrm{P}_{\mathrm{T}}=0.001\right)$, no statistically significant correlations between the PRS and the graph theoretical metrics of the visual subnetwork survived multiple-comparison correction. The following correlations, however, did survive multiple comparison correction:

The mean nodal strength of the NS-weighted visual subnetwork was correlated with the genome-wide PRS, including $A P O E$, for $\mathrm{P}_{\mathrm{T}}=0.1,0.3$ and 0.5 . The correlation coefficients were $r=-0.17,-0.18$ and -0.19 , for the 3 values of $P_{T}$ respectively, while the $p$-values were $8.4 \times 10^{-5}$, $4.1 \times 10^{-5}$ and $1.3 \times 10^{-5}$ respectively. When the analysis was repeated with the APOE locus excluded, the correlations persisted. Specifically, the correlation coefficients were: $-0.15,-0.17$ and -0.18 , while the $p$-values were $7.6 \times 10^{-4}, 1.4 \times 10^{-4}, 2.9 \times 10^{-5}$, for the 3 values of $P_{T}$ respectively. The correlations also persisted when the analysis was repeated for NS thresholds between 1 and 12 .

The mean clustering coefficient of the NS-weighted visual subnetwork was correlated with the tau protein binding PRS, including $A P O E$, for $\mathrm{P}_{\mathrm{T}}=0.3$ and 0.5 . The correlation coefficients were $r=-0.14$, while the $p$-values were $1.4 \times 10^{-3}$ for both $\mathrm{P}_{\mathrm{T}} \mathrm{s}$. When the analysis was repeated with the $A P O E$ locus excluded, the significance of the correlations disappeared, with the correlation coefficients being -0.02 and the $p$-values being 0.71 . The correlations persisted when the analysis was repeated for NS thresholds between 1 and 12 .

The mean betweenness centrality of the NS-weighted visual subnetwork was correlated with the plasma lipoprotein particle assembly PRS, including $A P O E$, for $\mathrm{P}_{\mathrm{T}}=0.3$ and 0.5 . The correlation coefficients were $r=0.15$ and 0.16 , for the 2 values of $P_{T}$ respectively, while the $p$ values were $9.2 \times 10^{-4}$ and $3.6 \times 10^{-4}$ respectively. When the analysis was repeated with the $A P O E$ locus excluded, the correlations persisted, with the correlation coefficients being $r=$ 0.12 and 0.13 for the two values of $P_{\mathrm{T}}$ respectively, and the $p$-values being $7.5 \times 10^{-3}$ and $2.2 \times 10^{-3}$ respectively. The correlations also persisted when the analysis was repeated for NS thresholds between 1 and 12 .

Repeating the analyses for the DMN using the first ten principal components derived from common alleles as covariates did not change these results.

All these results are shown in Fig. 6. 

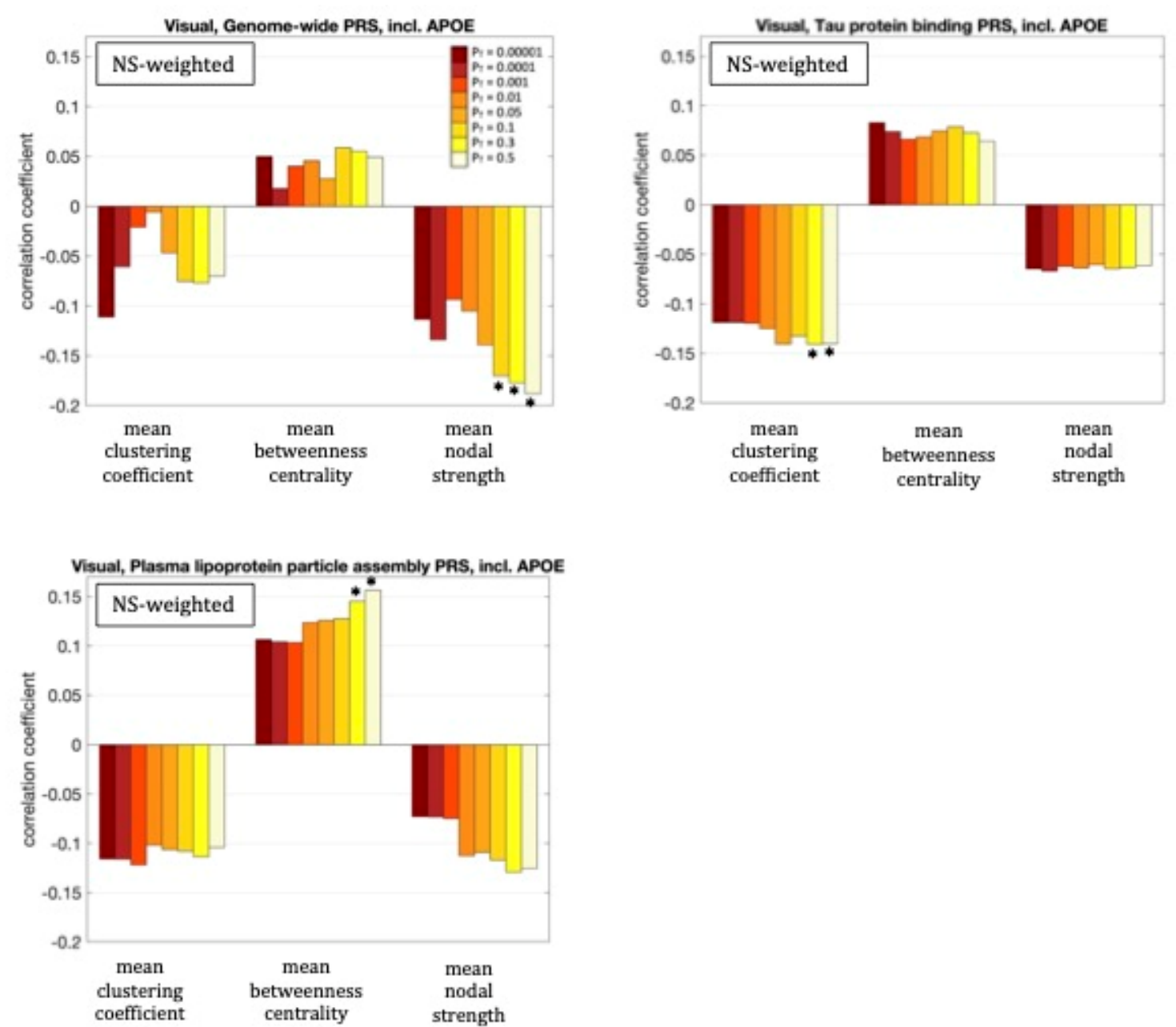

Figure 6: Correlation coefficients between the graph theoretical metrics of the visual subnetwork and the three PRSs for which those survived multiple comparison correction, for the 8 different values of $\mathrm{P}_{\mathrm{T}}$. The asterisk indicates the instances in which the $p$-value survived multiple comparison correction.

\subsection{Rich-club, feeder and local connectivity of the whole-brain network}

Fig. 7 shows the nodes that are hubs for the NS-weighted and the FA-weighted networks. For the NS-weighted networks, the hubs were the left and right putamen, left and right precuneus, left and right hippocampus, left and right superior frontal gyrus, left middle occipital gyrus, left and right superior occipital gyrus, right calcarine sulcus and right caudate. For the FAweighted networks, the hubs were the left and right putamen, left and right precuneus, left and right hippocampus, left and right superior frontal gyrus, left middle occipital gyrus, left calcarine sulcus, right superior parietal gyrus, left superior orbitofrontal gyrus and left superior occipital gyrus. We note that ten out of the 13 hubs were the same in the NS- and FAweighted networks, while three differed. 

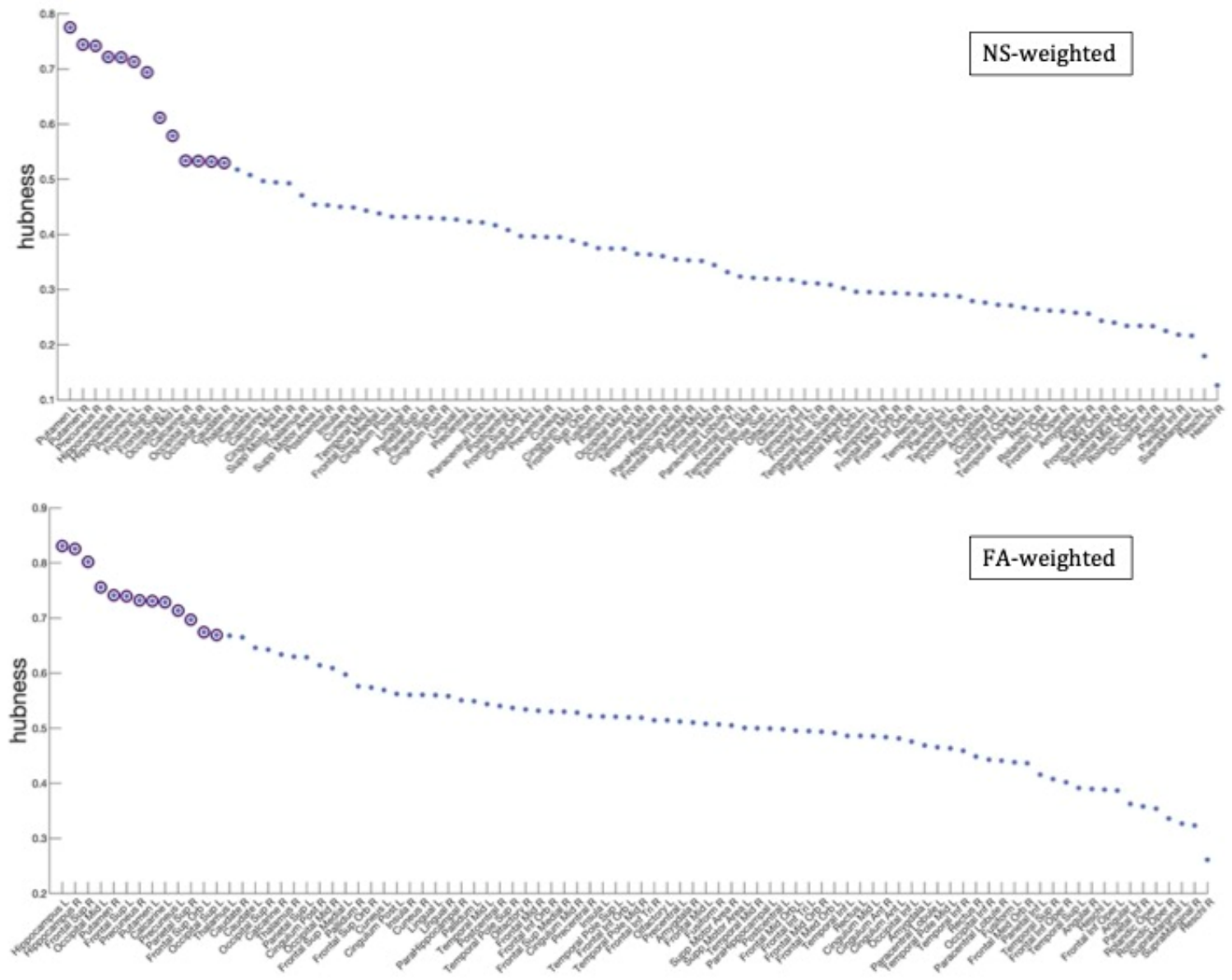

Figure 7: Hubness scores for the network nodes for the NS-weighted (top) and FA-weighted (bottom) networks. The purple circles indicate nodes that are hubs for the respective networks.

For our primary analysis $\left(\mathrm{P}_{\mathrm{T}}=0.001\right)$, no statistically significant correlations between the PRS and the rich-club, feeder or local connectivities of the whole-brain network survived multiplecomparison correction. The following correlations, however, did survive multiple comparison correction:

The rich-club connectivity of the NS-weighted whole-brain connectome was correlated with the genome-wide PRS, including $A P O E$, for $\mathrm{P}_{\mathrm{T}}=0.3$ and 0.5 . The correlation coefficients were $r=-0.16$ and -0.15 for the two $P_{T} S$ respectively, while the $p$-values were $3.7 \times 10^{-4}$ and $1.1 \times 10^{-3}$ respectively. When the analysis was repeated with the $A P O E$ locus excluded, the correlations persisted, with the correlation coefficients being $r=-0.15$ and -0.14 for the two $P_{T} S$ respectively, and the $p$-values being $6 \times 10^{-4}$ and $1.7 \times 10^{-3}$ respectively. The correlations also persisted when the analysis was repeated for NS thresholds of 1 to 12 .

The feeder connectivity of the NS-weighted whole-brain connectome was correlated with the genome-wide PRS, including $A P O E$, for $\mathrm{P}_{\mathrm{T}}=0.3$ and 0.5 . The correlation coefficients were $r=-0.14$ and -0.15 for the two $P_{T} S$ respectively, while the $p$-values were $1.3 \times 10^{-3}$ and $8.8 \times 10^{-4}$ respectively. When the analysis was repeated with the $A P O E$ locus excluded, the correlations persisted, with the correlation coefficients being $r=-0.14$ and -0.13 for the two $P_{T S}$ respectively, and the $p$-values being $1.4 \times 10^{-3}$ and $2.3 \times 10^{-3}$ respectively. The correlations also persisted when the analysis was repeated for NS thresholds of 1 to 12 . 
All these results are shown in Fig. 8.

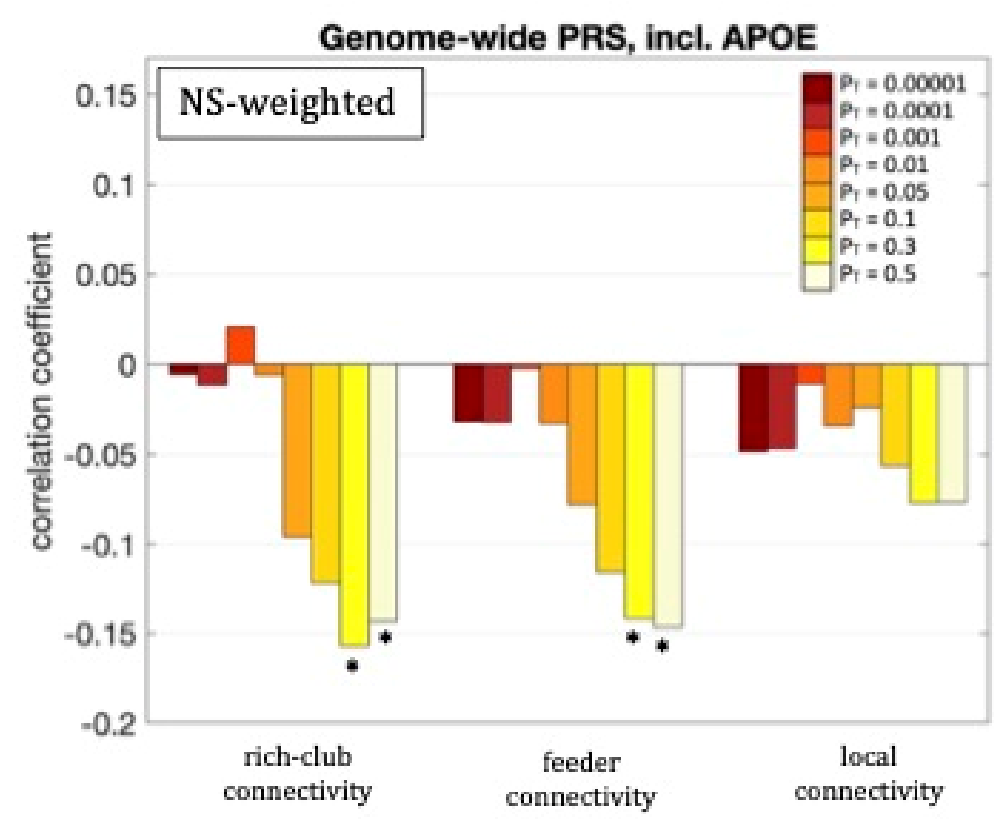

Figure 8: Correlation coefficients between the rich-club, feeder and local connectivities and the genome-wide PRS including $A P O E$, for the 8 different values of $\mathrm{P}_{\mathrm{T}}$. Asterisks indicate $p$-values that survived multiple comparison correction.

\section{Discussion}

To the best of our knowledge, this is the first study to examine the relationship between AD PRS and network-based measures for the whole-brain structural connectome and subnetworks. We used a cohort of young participants to assess any potential early changes in the structural connectome. From a clinical perspective, using pathway-specific polygenic risk scores in addition to genome-wide ones is important, because it can pave the way for more targeted interventions based on the predicted pathway involvement and potentially allow clinical trials to stratify patients using their specific risk profiles.

Compared to the FA-weighted networks, using NS-weighted networks resulted in more statistically significant relationships between the PRS and structural network metrics, such as the graph theoretical metrics we employed and the connectivity strength between the richclub, and feeder connections. Even though both the NS and the FA are routinely used to assign significance to the edges of structural networks, it has been argued (Huang and Ding, 2016) and proven experimentally (Messaritaki et al., 2021) that the NS is more relevant from a functional perspective to the network organization of the human brain compared to the FA. This may be contributing to the increased sensitivity of the NS in the differences observed in our study. From a methodological point of view, this demonstrates that the selection of the metric for the edge weights can impact the results and, if not optimal, it can fail to reveal certain statistically significant relationships.

Our analysis identified statistically significant (after correction for multiple comparisons) correlations between graph theoretical metrics and PRS, present in the DMN. The negative correlation between the mean nodal strength and the genome-wide PRS for the NS-weighted $D M N$ indicates that high genome-wide risk of $A D$ results in lower nodal strength in that network. Furthermore, the fact that the correlation persisted when the APOE locus was 
removed from the analysis indicates that this relationship is a result of multiple genetic factors and not exclusively due to the APOE gene.

Our analysis also revealed statistically significant (after multiple-comparison correction) correlations between the graph theoretical metrics of the NS-weighted visual subnetwork and the PRSs. The negative correlation between the mean nodal strength and the genome-wide PRS (including $A P O E$ ) implies weaker connectivity in the visual subnetwork of participants at higher risk of developing $A D$. The negative correlation between the mean clustering coefficient and the tau protein binding PRS (including $A P O E$ ) indicates that participants at higher risk of developing $A D$ through this pathway have less clustered communities in the visual subnetwork. The positive correlation between the mean betweenness centrality and the PRS for plasma lipoprotein particle assembly (including $A P O E$ ) implies that, in participants at higher risk of developing $A D$, each node participates in more shortest paths and therefore the organisation of the visual subnetwork is less central compared to participants at low risk. The fact that the first and third of these correlations persisted when the APOE locus was excluded from the genetic risk calculation indicates that they are a result of multiple genetic factors, and not exclusively due to the APOE gene. The second correlation, however, appears to be driven predominantly by the $A P O E$ gene.

As mentioned earlier, alterations in the visual subnetwork of $A D$ patients have been recently reported in the literature. For example, Deng et al. (2016) observed increased characteristic path length and clustering coefficient in the visual subnetwork (measured with BOLD fMRI) of AD patients. Badhwar et al. (2017) also observed decreased connectivity in the primary visual cortex of AD patients. Wang et al. (2019) observed impairments in the visual subnetwork of $A D$ patients, as well as in patients with subjective cognitive decline, which is considered a prodromal stage of $A D$. This last result further supports the idea that alterations in the visual subnetwork can appear many years before AD diagnosis.

We also observed statistically significant correlations (after multiple-comparison correction) between the rich-club and feeder connectivities of the NS-weighted whole-brain network and the genome-wide PRS, including APOE. These negative correlations indicate that structural connections that involve at least one hub node are weaker in the brains of young participants at risk of developing AD. The relationships held when the APOE locus was excluded from the analysis, which indicates that the effect comes from genetic influences above and beyond APOE.

A few studies have reported altered connectivity of the rich-club and feeder edges in the structural brain networks of participants with Alzheimer's disease and with mild cognitive impairment. Xue et al. (2020) recently observed reduced rich-club connectivity in patients with amnestic $\mathrm{MCl}$ compared to healthy age-matched controls, and reduced feeder and local connectivity in patients with amnestic $\mathrm{MCl}$ compared to participants with subjective cognitive decline. Cai et al. (2019) reported decreased feeder (and local) connection strength in the structural networks of AD patients compared to healthy controls. Our results are in line with these alterations in connectivity strength observed in $A D$ and $\mathrm{MCl}$ patients.

The rest of the graph theoretical metrics we investigated showed no statistically significant correlations after multiple comparison correction was applied. Recently, Foley et al. (2017) showed that there is a reduction in the fractional anisotropy of the right cingulum and a decrease in the left hippocampal volume of young adults at genetic risk of developing AD. In this context, our results imply that those alterations do not translate into changes in the structural brain networks and subnetworks of those young adults. We note, however, that the participants in that study included, in addition to participants of the age of those in our study, participants that were a few years older. 
The correlations observed in our analysis are small. This is to be expected, given that the cohort of our study consisted of young adults with normal brain function.

We note that the summary statistics used in PRS analysis were taken from a large discovery sample reported in the latest GWAS meta-analysis (Kunkle et al., 2019). Therefore, our risk estimates for AD loci are the best available. Our study employed a relatively large sample size comprising participants of the same age $(19.81 \pm 0.02$ years old), therefore avoiding the confound of brain changes that are age related, and which are known to exist in young adults up to the age of at least 25 years. Furthermore, our study is the first to use disease pathway PRS to explore associations between biological pathways and underlying differences in structural brain connectivity. Regarding the pathway-specific PRS, the accuracy of our results is limited by the current knowledge of pathway variants. We note that our study involved a geographically limited sample in which men are slightly over-represented. Therefore, our results may not be representative of the general population.

\section{Conclusion}

Our results demonstrate that genetic burden is linked to changes in structural brain networks, both for the whole-brain connectome and the visual subnetwork, in young adults. The genome-wide PRS including $A P O E$ was linked to a reduction in the mean nodal strength of the visual subnetwork and of the rich-club connections of the whole-brain network. The plasma-lipoprotein particle assembly PRS including APOE was linked to an increase in the betweenness centrality of the visual subnetwork. Importantly, these relationships were still present, albeit slightly weaker, when the APOE locus was excluded from the analysis. This indicates that the search for $A D$ biomarkers can benefit from the consideration of genetic risk above and beyond $A P O E$. Different biomarkers could point to different pathway involvement, which could allow clinical trials to stratify patients accordingly.

Note: EM and JRH are both last authors of the article.

\section{Acknowledgements}

We are extremely grateful to the families that took part in this study, the midwives for their help in recruiting them, and the whole ALSPAC team, which includes interviewers, computer and laboratory technicians, clerical workers, research scientists, volunteers, managers, receptionists and nurses. We also wish to thank Dr. Maryam Afzali for useful discussions.

\section{Funding}

The UK Medical Research Council and Wellcome (Grant ref: 217065/Z/19/Z) and the University of Bristol provide core support for ALSPAC. This publication is the work of the authors and Mirza-Davies et al. will serve as guarantors for the contents of the paper. This research was funded in whole, or in part, by the Wellcome Trust (Grant numbers: 204824/Z/16/Z, 096646/Z/11/Z, 104943/Z/14/Z, 203918/Z/16/Z). For the purpose of Open Access, the author has applied a CC BY public copyright license to any Author Accepted Manuscript version arising from this submission. A comprehensive list of grants funding is available on the ALSPAC website (http://www.birstol.ac.uk/alspac/external/documents/grantacknowledgements.pdf). EM was partly funded by a Wellcome Trust ISSF Postdoctoral Research Fellowship at Cardiff University (204824/Z/16/Z). EM was also supported by the 
BRAIN Biomedical Research Unit which is funded by Health and Care Research Wales. DKJ was funded by a Wellcome Trust Investigator Award (096646/Z/11/Z) and a Wellcome Trust Strategic Award (104943/Z/14/Z). JRH was funded by a Wellcome Trust GW4 Clinical Academic Fellowship (203918/Z/16/Z). SF was funded on an Institutional Strategic Support Fund Grant No. 504182 awarded to Cardiff University by the Wellcome Trust. PH was funded by a Medical Research Council Award (MR/L010305/1) at the MRC Centre for Neuropsychiatric Genetics \& Genomics at Cardiff University. VEP and EB were funded by the Dementia Research Institute [UKDRI supported by the Medical Research Council (UKDRI3003), Alzheimer's Research UK, and Alzheimer's Society], the Welsh Government, Joint Programming for Neurodegeneration (MRC: MR/T04604X/1), Dementia Platforms UK (MRC: MR/L023784/2), and MRC Centre for Neuropsychiatric Genetics and Genomics (MR/L010305/1).

\section{References}

M. Afzali, H. Knutsson, E. Ozarslan, D. K. Jones. "Computing the orientational-average of diffusion-weighted MRI signals: a comparison of different techniques.", Scientific Reports, $2021,11: 14345$.

G. Allen, H. Barnard, R. McColl, A.L. Hester, J.A. Fields, M.F. Weiner, W.K. Ringe, A.M. Lipton. M. Brooker, E. McDonald, C.D. Rubin, M. Cullum. "Reduced hippocampal functional connectivity in Alzheimer's disease." Arch Neurol, 2007, 64(10):1482-1487. doi:10.1001/archneur.64.10.1482

A. Altmann, M. A. Scelsi, M. Shoai, E. de Silva, L. M. Aksman, D. M. Cash, J. Hardy, J. M. Schott. "A comprehensive analysis of methods for assessing polygenic burden in Alzheimer's disease pathology and risk beyond APOE." Brain Commun., 2020, 2(1):fcz047.

A. Arnatkeviciute, B.D. Fulcher, S. Oldham, J. Tiego, C. Paquola, Z. Gerring, K. Aquino, Z. Hawi, B. Johnson, G. Ball, M. Klein, G. Deco, B. Franke, M. Bellgrove, A. Fornito. "Genetic influences on hub connectivity of the human connectome." Nat Commun 12, 4237 (2021).

A.P. Badhwar, A. Tam, C. Dansereau, P. Orban, F. Hoffstaedter, P. Bellec. "Resting-state network dysfunction in Alzheimer's disease: A systematic review and meta-analysis." Alzheimer's and Dementia: Diagnosis, Assessment and Disease Monitoring, 2017, 8, 73-85. doi.org/10.106/j.dadm.2017.03.007

Y. Benjamini and D. Yekutieli. "False discover rate-adjusted multiple confidence intervals for selected parameters." Journal of the American Statistical Association, 2005, 100(469):71-81. doi:10.1198/016214504000001907

R.F. Betzel, L. Byrge, Y. He, J. Goni, X.-N. Zuo, O. Sporns. "Changes in structural and functional connectivity among resting-state networks across the human lifespan." Neurolmage, 2014, 102(2):345-357. https://doi.org/10.1016/j.neuroimage.2014.07.067

A. Boyd, J. Golding, J. Macleod, D.A. Lawlor, A. Fraser, J. Henderson, L. Molloy, A. Ness, S. Ring, G. Davey Smith. "Cohort Profile: the 'children of the 90s'--the index offspring of the Avon Longitudinal Study of Parents and Children." Int J Epidemiol. 2013, 42(1):111-127. doi:10.1093/ije/dys064

J.A. Brown, K.H. Terashima, A.C. Burggren, L.M. Ercoli, K.J. Miller, G.W. Small, S.Y. Bookheimer. "Brain network local interconnectivity loss in aging APOE4 allele carriers." Proc Natl Acad Sci U S A, 2011, 108(51):20760-20765. doi:10.1073/pnas.1109038108 
S. Cai, K. Huang, Y. Kang, Y. Jiang, K.M. von Deneen, L. Huang. "Potential biomarkers for distinguishing people with Alzheimer's disease from cognitively intact elderly based on the rich-club hierarchical structure of white matter networks." Neuroscience Research, 2019, 144:56-66.

S. Caspers, M. E. Rockner, C. Jockwitz, N. Bittner, A. Teumer, S. Herms, P. Hoffmann, M. Nothen, S. Moebus, K. Amunts, S. Cichon, T. W. Muhleisen. "Pathway-specific genetic risk for Alzheimer's disease differentiates regional patterns of cortical atrophy in older adults." Cereb Cortex, 2020, 30(2):801-811.

C.C Chang, C.C. Chow, L.C. Tellier, S. Vattikuti, S.M. Purcell, J.J. Lee. "Second-generation PLINK: rising to the challenge of larger and richer datasets." Gigascience, 2015, 4:7. doi:10.1186/s13742-015-0047-8

L.C. Chang, D.K. Jones, C. Pierpaoli. "RESTORE: robust estimation of tensors by outlier rejection." Magn Reson Med. 2005, 53(5):1088-1095. doi:10.1002/mrm.20426

S. Chaudhury, T. Patel, I.S. Barber, et al. "Polygenic risk score in postmortem diagnosed sporadic early-onset Alzheimer's disease." Neurobiol Aging, 2018, 62:244 e1-244.e8. doi:10.1016/j.neurobiolaging.2017.09.035

H.J. Clarke, E. Messaritaki, S.I. Dimitriadis, C. Metzler-Baddeley. "Dementia risk factors modify hubs but leave other connectivity measures unchanged in asymptomatic individuals: a graph theoretical analysis." 2020, Brain Connectivity, https://doi.org/10.1089/brain.2020.0935.

R.D. Cook. "Detection of influential observations in linear regression." 1977, Technometrics, 19(1):15-18.

F. Corlier, G. Hafzalla, J. Faskowitz, L.H. Kuller, J.T. Becker, O.L. Lopez, P.M. Thompson, M.M. Braskie. "Systemic inflammation as a predictor of brain aging: Contributions of physical activity, metabolic risk, and genetic risk." Neuroimage, 2018, 172:118-129. doi:10.1016/j.neuroimage.2017.12.027

C. Cruchaga, J.L Del-Aguila, B. Saef et al. "Polygenic risk score of sporadic late-onset Alzheimer's disease reveals a shared architecture with the familial and early-onset forms." Alzheimers Dement, 2018, 14(2):205-214. doi:10.1016/j.jalz.2017.08.013

Z. Dai, Q. Lin, T. Li, X. Wang, H, Yuan, X. Yu, Y. He, H. Wang. "Disrupted structural and functional brain networks in Alzheimer's disease." Neurobiol Aging, 2019, 75:71-82. doi:10.1016/j.neurobiolaging.2018.11.005

C.A. de Leeuw, J.M. Mooij, T. Heskes, D. Posthuma. "MAGMA: generalized gene-set analysis of GWAS data." PLos Comput Biol. 2015, 11(4):e1004219. doi:10.1371/journal.pcbi.1004219

F. Dell'acqua, P. Scifo, G. Rizzo, M. Catani, A. Simmons, G. Scotti, F. Fazio. "A modified damped Richardson-Lucy algorithm to reduce isotropic background effects in spherical deconvolution." Neurolmage. 2010, 49(2):1446- 1458. doi:10.1016/j.neuroimage.2009.09.033

Y. Deng, L. Shi, Y. Li, D. Wang, Alzheimer's Neuroimaging Initiative. "Altered topological organization of high-level visual networks in Alzheimer's disease and mild cognitive impairment patients." Neuroscience Letters. 2016, 630:147-153. 
N.A. Dennis, J.N. Browndyke, J. Stokes, A. Need, J.R. Burke, K.A. Welsh-Bohmer, R. Cabeza. "Temporal lobe functional activity and connectivity in young adult APOE4 carriers." Alzheimers Dement, 2010, 6(4):303-311. doi:10.1016/j.jalz.2009.07.003

S. Dimitriadis, E. Messaritaki, D. K. Jones. "The impact of graph construction scheme and community detection algorithm on the repeatability of community and hub identification in structural brain networks." Human Brain Mapping, 2021, 42(13):4261-4280.

M. Drakesmith, K. Caeyenberghs, A. Dutt, S. Zammit, C. J. Evans, A. Reichenberg, G. Lewis, A. S. David, D. K. Jones. "Schizophrenia-like topological changes in the structural connectome of individuals with subclinical psychotic experiences." Human Brain Mapping, 2015, 36(7):2629-2643.

M. Drakesmith, A. Dutt, L. Fonville, S. Zammit, A. Reichenberg, C. J. Evans, P. McGuire, G. Lewis, D. K. Jones, A. S. David. "Volumetric, relaxometric and diffusometric correlates of psychotic experiences in a non-clinical sample of young adults." Neurolmage: Clinical, 2016, 12:550-558.

M. Drakesmith, G. D. Parker, J. Smith, S. C. Linden, E. Rees, N. Williams, M. J. Owen, M. van den Bree, J. Hall, D. K. Jones, D. E. J. Linden. "Genetic risk for schizophrenia and developmental delay is associated with shape and microstructure of midline white matter structures." Translational Psychiatry, 2019, 9:102.

V. Escott-Price, R. Sims, C. Bannister, et al. "Common polygenic variation enhances risk prediction for Alzheimer's disease." Brain, 2015, 138(12):3673-3684. doi.org/10.1093/brain/awv268

V. Escott-Price, A.J. Myers, M. Huentelman, J. Hardy. "Polygenic risk score analysis of pathologically confirmed Alzheimer's disease." Ann Neurol, 2017, 82(2):311-314. doi:10.1002/ana.24999

L.A. Farrer, L.A. Cupples, J. L. Haines, B. Hyman, W.A. Kukull, R. Mayeux, R.H. Myers, M.A. Pericak-Vance, N. Risch, C.M. van Duijn. "Effects of age, sex, and ethnicity on the association between apolipoprotein $\mathrm{E}$ genotype and Alzheimer's disease. A meta-analysis. APOE and Alzheimer Disease Meta Analysis Consortium." JAMA, 1997, 278(16):1349-1356

N. Filippini, B.J. Machintosh, M.G. Hough, G.M. Goodwin, G.B. Frisoni, S.M. Smith, P.M. Matthews, C.F. Beckmann, C.E. Mackay. "Distinct patterns of brain activity in young carriers of the APOE-epsilon4 allele." Proc Natl Acad Sci USA, 2009, 106(17):7209-7214. doi:10.1073/pnas.0811879106

A.S. Fleisher, A. Sherzai, C. Taylor, J.B. Langbaum, K. Chen, R.B. Buxton. "Resting-state BOLD networks versus task-associated functional MRI for distinguishing Alzheimer's disease risk groups." Neurolmage, 2009, 47(4):1678-1690. doi:10.1016/j.neuroimage.2009.06.021

S.F. Foley, K.E. Tansey, X. Caseras, T. Lancaster, T. Bracht, G. Parker, J. Hall, J. Williams, D.E.J. Linden. "Multimodal Brain Imaging Reveals Structural Differences in Alzheimer's Disease Polygenic Risk Carriers: A Study in Healthy Young Adults." Biol Psychiatry, 2017, 81(2):154-161. doi:10.1016/j.biopsych.2016.02.03322

S.F. Foley, M. Bracher-Smith, K.E. Tansey, J.R. Harrison, G.D. Parker, X. Caseras. "Fractional anisotropy of the uncinate fasciculus and cingulum in bipolar disorder type I, type II, unaffected siblings and healthy controls." $B r J$ Psychiatry. 2018, 213(3):548-554. doi:10.1192/bjp.2018.101 
L. Fonville, K. Cohen Kadosh, M. Drakesmith, A. Dutt, S. Zammit, J. Mollon, A. Reichenberg, G. Lewis, D. K. Jones, A. S. David. "Psychotic experiences, working memory, and the developing brain: a multimodal neuroimaging study." Cerebral Cortex, 2015, 25(12):48284838.

A. Fraser, C. Macdonald-Wallis, K. Tilling, A. Boyd, J. Golding, G. Davey Smith, J. Henderson, J. Macleod, L. Molloy, A. Ness, S. Ring, S.M. Nelson, D.A. Lawlor. "Cohort Profile: the Avon Longitudinal Study of Parents and Children: ALSPAC mothers cohort." Int J Epidemiol. 2013, 42(1):97-110. doi:10.1093/ije/dys066

M. Gatz, C.A. Reynolds, L. Fratiglioni, B. Johansson, J.A. Mortimer, S. Berg, A. Fiske, N.L. Pedersen. "Roles of genes and environments for explaining Alzheimer's disease." Arch Gen Psychiatry, 2006, 63(2):168-174. doi:10.1001/archpsyc.63.2.168

J. Goni, M.P. van den Heuvel, A. Avena-Koenigsberger, N. Veles de Mendizabal, R.F. Betzel, A. Griffa, P. Hagmann, B. Corominas-Murtra, J.-P. Thiran, O. Sporns. "Resting-brain functional connectivity predicted by analytic measures of network communication." PNAS, 2014, 111(2):833-838. doi.org/10.1073/pnas.1315529111

J.S. Goveas, C. Xie, G. Chen, W. Li, B.D. Ward, M.B. Franczak, J.L. Jones, P.G. Antuono, S.J. Li. "Functional network endophenotypes unravel the effects of apolipoprotein E epsilon 4 in middle-aged adults." PLoS One, 2013, 8(2):e55902. doi:10.1371/journal.pone.0055902

O. Hansson, M. J. Grothe, T. O. Strandberg, T. Ohlsson, D. Hagerstrom, J. Jogi, R. Smith and M. Scholl for the Alzheimer's Disease Neuroimaging Initiative and for the Swedish BioFINDER Study. "Tau pathology distribution in Alzheimer's disease corresponds differentially to cognition-relevant functional brain networks." Front Neurosci, 2017, doi.org/10.3389/fnins.2017.00167.

J.R. Harrison, S. Mistry, N. Muskett, V. Escott-Price. "From polygenic scores to precision medicine in Alzheimer's disease: A systematic review." J Alzheimers Dis, 2020, 10.3233/JAD191233. doi:10.3233/JAD-191233

T. M. Harrison, Z. Mahmood, E. P. Lau, A. M. Karacozoff, A. C. Burggren, G. W. Small, S. Y. Bookheimer. "An Alzheimer's disease genetic risk score predicts longitudinal thinning of hippocampal complex subregions in healthy older adults." eNeuro, 2016;3(3):795-804. doi:10.1523/ENEURO.0098-16.2016

J. P. Hayes, M. W. Logue, N. Sadeh, J. M. Spielberg, M. Verfaellie, S. M. Hayes, A. Reagan, D. H. Salat, E. J. Wolf, R. E. McGlinchey, W. P. Milberg, A. Stone, S. A. Schichman, M.W. Miller. "Mild traumatic brain injury is associated with reduced cortical thickness in those at risk of Alzheimer's disease." Brain, 2017, 140(3):813-825.

Y. He, Z. Chen, A. Evans. "Structural insights into aberrant topological patterns of large-scale cortical networks in Alzheimer's disease." J Neurosci, 2008, 28(18):4756-4766. doi:10.1523/JNEUROSCI.0141-08.2008

C.J. Honey, O, Sporns, L. Cammoun, X. Gigandet, J.P. Thiran, R. Meuli, P. Hagmann. "Predicting human resting-state functional connectivity from structural connectivity." PNAS, 2009, 106(6):2035-2040. doi.org/10.1073/pnas.0811168106

H. Huang and M. Ding. "Linking functional connectivity and structural connectivity quantitatively: a comparison of methods." Brain Connectivity, 2016, 6(2): 99-108. 
M.O. Irfanoglu, L. Walker, J. Sarlls, S. Marenco, C. Pierpaoli. "Effects of image distortions originating from susceptibility variations and concomitant fields on diffusion MRI tractography results." Neuroimage. 2012, 61(1):275-288. doi:10.1016/j.neuroimage.2012.02.054

M. John, T. Ikuta, J. Ferbinteanu. "Graph analysis of structural brain networks in Alzheimer's disease: beyond small world properties." Brain Struct Funct, 2017, 222(2):923-942. doi:10.1007/s00429-016-1255-4

D.K. Jones, M.A. Horsfield, A. Simmons. "Optimal strategies for measuring diffusion in anisotropic systems by magnetic resonance imaging. Magn Reson Med. 1999, 42(3):515-525

L. Jones, P.A. Holmans, M.L. Hamshere et al. "Genetic evidence implicates the immune system and cholesterol metabolism in the aetiology of Alzheimer's disease." PLoS One, 2010, 5(11):e13950. https://doi.org/10.1371/journal.pone.0013950

B.W. Kunkle, B. Grenier-Boley, R. Sims, et al. "Genetic meta-analysis of diagnosed Alzheimer's disease identifies new risk loci and implicates $A \beta$, tau, immunity and lipid processing." Nat Genet 2019, 51(3): 414-430. doi:10.1038/s41588-019-0358-2

J.C. Lambert, C.A. Ibrahim-Verbaas, D. Harold et al. "Meta-analysis of 74,046 individuals identifies 11 new susceptibility loci for Alzheimer's disease." Nat Genet, 2013, 45(12):14521458. doi:10.1038/ng.2802

T. M. Lancaster, S. Dimitriadis, K. E. Tansey, G. Perry, N. Ihssen, D. K. Jones, K. D. Singh, P. Holmans, A. Pocklingtom, G. Davey Smith, S. Zammit, J. Hall, M. C. O;Donovan, M. J. Owen, D. E. Linden. 'Structural and functional neuroimaging of polygenic risk for schizophrenia: a recall-by-genotype-based approach." Schizophr Bull., 2019, 45(2):405-414.

D. Le Bihan, C. Poupon, A. Amadon, F. Lethimonnier. "Artifacts and pitfalls in diffusion MRI" Journal of Magnetic Resonance Imaging, 2006, 24:478-488.

A. Leemans, D.K. Jones. "The B-matrix must be rotated when correcting for subject motion in DTI data." Magn Reson Med. 2009, 61(6):1336-1349. doi:10.1002/mrm.21890

A. Leemans, B. Jeurissen, J. Sijbers, D.K. Jones. "ExploreDTI: A graphical toolbox for processing, analysing and visualizing diffusion MRI data.", $17^{\text {th }}$ Annual Meeting of the International Society for Magnetic Resonance in Medicine, Hawaii, USA, vol. 3537, 2009

J. Li, X. Zhang, A. Li, S. Liu, W. Qin, C. Yu, Y. Liu, B. Liu, T. Jiang. "Polygenic risk for Alzheimer's disease influences precuneal volume in two independent general populations." Neurobiol Aging, 2018, 64:116-122. doi:10.1016/j.neurobiolaging.2017.12.022

Z. Liao, Y. Patel, A. Khairullah, N. Parker, T. Paus. "Pubertal testosterone and the structure of the cerebral cortex in young men." Cerebral Cortex, 2021, 31(6):2812-2821.

C.Y. Lo, P.N. Wang, K.H. Chou, J. Wang, Y. He, C.P. Lin. "Diffusion tensor tractography reveals abnormal topological organization in structural cortical networks in Alzheimer's disease." J Neurosci, 2010, 30(50):16876-16885. doi:10.1523/JNEUROSCI.4136-10.2010

M. K. Lupton, L. Strike, N. K. Hansell, W, Wen, K. A. Mather, N. J. Armstrong, A. Thalamuthu, K. L. McMahon, G. I. de Zubicaray, A. A. Assareh, A. Simmons, P. Proitsi, J. F. Powell, G. W. Montgomery, D. P. Hibar, E. Westman, M. Tsolaki, I. Kloszewska, H. Soininen, P. Mecocci, B. Velas, S. Lovestone, Alzheimer's disease Neuroimaging Initiative, H. Brodaty, D. Ames, J. N. Trollor, N. G. Martin, P. M. Thompson, P. S. Sachdeb, M. J. Wright. "The effect of increased 
genetic risk for Alzheimer's disease on hippocampal and amygdala volume." Neurobiol. Aging, 2016, 40:68-77.

C. Ma, J. Wang, J. Zhang, K. Chen, X. Li, N. Shu, Y. Chen, Z. Liu, Z. Zhang. "Disrupted Brain Structural Connectivity: Pathological Interactions Between Genetic APOE $\varepsilon 4$ Status and Developed MCl Condition." Mol Neurobiol, 2017, 54(9):6999-7007. doi:10.1007/s12035-0160224-5

E. Messaritaki, S.I. Dimitriadis, D.K. Jones. "Optimization of graph construction can significantly increase the power of structural brain network studies." Neurolmage, 2019, 199:495-511, doi.org/10.1016/j.neuroimage.2019.05.052

E. Messaritaki, S. Foley, S. Schiavi, L. Magazzini, B. Routley, D. K. Jones and K.D. Singh. "Predicting MEG resting-state functional connectivity from microstructural information." Network Neuroscience, 2021, 5(2):477-504.

R. Mito, D. Raffelt, T. Dhollander, D.N. Vaughan, J.-D. Tournier, O. Salvado, A. Brodtmann, C.C. Rowe, V.L. Villemagne, A. Connely. "Fibre-specific white matter reductions in Alzheimer's disease and mild cognitive impairment." Brain, 2018, 141(3):888-902. doi:10.1093/brain/awx355

A. Mohan, A.J. Roberto, A. Mohan, A. Lorenzo, K. Jones, M.J. Carney, L. Liogier-Weyback, S. Hwang, K.A.B. Lapidus. "The Significance of the Default Mode Network (DMN) in Neurological and Neuropsychiatric Disorders: A Review." Yale J Biol Med, 2016, 89(1):49- 57

E.C. Mormino, R.A. Sperling, A.J. Holmes, R.L. Buckner, P.L. De Jager, J.W. Smoller, M.R. Sabuncu, For the Alzheimer's Disease Neuroimaging Initiative. "Polygenic risk of Alzheimer disease is associated with early- and late-life processes." Neurology, 2016, 87(5):481-488. doi:10.1212/WNL.0000000000002922

K. Northstone, M. Lewcock, A. Groom, A. Boyd, J. Macleod, N. J. Timpson, N. Wells. "The Avon Longitudinal Study of Parents and Children (ALSPAC): an updated on the enrolled sample of index children in 2019." Wellcome Open Res, 2019, 4:51 doi.org/10.12688/wellcomeopenres.15132.1

S. Oldman and A. Fornito. "The development of brain network hubs." Developmental Cognitive Neuroscience, 2019, 36:100607. doi.org/10.1016/j.dcn.2018.12.005

G. Parker, D. Marshall, P. Rosin, N. Drage, S. Richmond, D.K. Jones. "RESDORE: robust estimation in spherical deconvolution by outlier rejection. Proceedings of the 21st Annual Meeting of ISMRM, Salt Lake City, Utah, USA, 2013a

G. Parker, D. Marshall, P.L. Rosin, N. Drage, S. Richmond, D.K. Jones. "Fast and fully automated clustering of whole brain tractography results using shape-space analysis." Proceedings of the 21st Annual Meeting of ISMRM, Salt Lake City, Utah, USA, 2013.

O. Pasternak, N. Sochen, Y. Gur, N. Intrator, Y. Assaf. "Free water elimination and mapping from diffusion MRI." Magn Reson Med. 2009, 62(3):717-730. doi:10.1002/mrm.22055

Y. Patel, J. Shin, M. Drakesmith, J. Evans, Z. Pausova, T. Paus. "Virtual histology of multimodal magnetic resonance imaging of cerebral cortex in young men." Neurolmage, 2020, 218:116968. 
J.D. Power, A.L. Cohen, S.M. Nelson, S.G. Wig, K. Anne, J.A. Church, A.C. Vogel, T.O. Laumann, F.M. Miezin. "Functional network organisation of the human brain." Neuron, 2011, 72(4) 665-678. Doi,org/10.1016/j.neuron.2011.09.006

A. Prestia, A. Caroli, K. Herholz, E. Reiman, K. Chen, W.J. Jagust et al. "Diagnostic accuracy of markers for prodromal Alzheimer's disease in independent clinical series." Alzheimers Dement. 2013, 9(6):677-686. doi:10.1016/j.jalz.2012.09.016

M. Prince, R. Bryce, E. Albanese, A. Wimo, W. Ribeiro, C.P. Ferri. "The global prevalence of dementia: a systematic review and meta-analysis.” Alzheimer's Dement. 2013, 9(1):63-75.

S.M. Purcell, N.R. Wray, J.L. Stone, P.M. Visscher, M.C. O’Donovan, P.F. Sullivan, P. Sklar; International Schizophrenia Consortium. "Common polygenic variation contributes to risk of schizophrenia and bipolar disorder." Nature, 2009, 460:748-752. doi:10.1038/nature08185

T. Roine, B. Jeurissen, D. Perrone, J. Aelterman, W. Phillips, J. Sijbers, A. Leemans. "Reproducibility and intercorrelation of graph theoretical measures in structural brain connectivity networks." Medical Image Analysis, 2019, 52:56-67. doi.org/10.1016/j.media.2018.10.009

J. Rowley, V. Fonov, O. Wu, S. Fristed Eskildsen, D. Schoemaker, L. Wu, S. Mohades, M. Shin, V. Sziklas, L. Cheewakriengkrai, A. Shmuel, A. Dagher, S. Gauthier, P. Rosa-Neto, for the Alzheimer's Disease Neuroimaging Initiative. "White matter abnormalities and structural hippocampal disconnections in amnestic mild cognitive impairment and Alzheimer's disease." PLoS One, 2013, doi.org/10.1371/journal.pone.0074776

M. Rubinov and O. Sporns. "Complex network measures of brain connectivity: uses and interpretations." Neurolmage, 2010, 52(3):1059-1069. Doi:10.1016/j.neuroimage.2009.10.003

M. R. Sabuncu, R. L. Buckner, J. W. Smoller, P. Hyoun Lee, B. Fischl, R. A. Sperling, Alzheimer's Disease Neuroimaging Initiative. "The association between a polygenic Alzheimer's score and cortical thickness in clinically normal subjects." Cereb Cortex, 2012, 22(11):2653-61.

T. H. Sharp, N. S. McBride, A. E. Howell, C. J. Evans, D. K. Jones, G. Perry, S. I. Dimitriadis, T. M. Lancaster, L. Zuccolo, C. Relton, S. M. Matthews, T. Breeze, A. S. David, M. Drakesmith, D. E. J. Linden, T. Paus, E. Walton. "Population neuroimaging: generation of a comprehensive data resource within the ALSPAC pregnancy and birth cohort." [version 1; peer review: 1 approved, 2 approved with reservations]. Wellcome Open Res 2020, 5:203. https://doi.org/10.12688/wellcomeopenres.16060.1

R. Sims, M. Hill, J. Williams. "The multiplex model of the genetics of Alzheimer's disease." Nat Neurosci, 2020, 23(3):311-322. doi:10.1038/s41593-020-0599-5

K. Sleegers, K. Bettens, A. De Roeck, et al. "A 22-single nucleotide polymorphism Alzheimer's disease risk score correlates with family history, onset age, and cerebrospinal fluid A $342 . "$ Alzheimers Dement, 2015, 11(12):1452-1460. doi:10.1016/j.jalz.2015.02.013

R.A. Sperling, P.S. Aisen, L.A. Beckett, D.A. Bennett, S. Craft, A.M. Fagan, et al. "Toward defining the preclinical stages of Alzheimer's disease: Recommendations from the National Institute of Aging-Alzheimer's Association workgroups on diagnostic guidelines for Alzheimer's disease." Alzheimer's Dement, 2011, 7:280-292. doi.org/10.1016/j.jalz.2011.03.003

R. E. Tanzi. "The genetics of Alzheimer's disease." Cold Spring Harb Perspect Med, 2012, 2(10): a006296. 
G. Tosto, T.D. Bird, D. Tsuang, D.A. Bennett, B.F. Boeve, C. Cruchaga, K. Faber, T.M. Foroud, M. Farlow, A.M. Goate, S. Bertlesen, N.R. Graff-Radford, M. Medrano, R. Lantigua, J. Manly, R. Ottman, R. Rosenberg, D.J. Schaid, N. Schupf, Y. Stern, R.A. Sweet, R. Mayeux. "Polygenic risk scores in familial Alzheimer disease." Neurology, 2017, 88(12):1180-1186. doi:10.1212/WNL.0000000000003734

N. Tzourio-Mazoyer, B. Landeau, D. Papathanassiou, F. Crivello, O. Etard, N. Delcroix, B. Mazoyer, M. Joliot. "Automated anatomical labeling of activations in SPM using a macroscopic anatomical parcellation of the MNI MRI single subject brain." Neuroimage, 2002, 15(1):273289. doi:10.1006/nimg.2001.0978

M.P. van den Heuvel and O. Sporns. "Rich-club organization of the human connectome." Journal of Neuroscience, 2011, 31(44): 15775-15786.

https://doi.org/10.1523/JNEUROSCI.3539-11.2011

L. Wang, Y. Zhang, Y. He, M. Liang, X. Zhang, L. Tian, T. Wu, T. Jiang, K. Li. "Changes in hippocampal connectivity in the early stages of Alzheimer's disease: Evidence from resting state fMRI." Neurolmage, 2006, 31(2):496-504. doi.org/10.1016/j.neuroimage.2005.12.033

Z. Wang, K. Qiao, G. Chen, D. Sui, H.-M. Dong, Y.-S. Wang, H.-J. Li, J. Lu, X.-N. Zuo, Y. Han. "Functional connectivity changes across the spectrum of subjective cognitive decline, amnestic mild cognitive impairment and Alzheimer's disease." Front Neuroinform, 2019. Doi: https://doi.org/10.3389/fninf.2019.00026

N.R. Wray, S.H. Lee, D. Mehta, A.A. Vinkhuyzen, F. Dudbridge, C.M. Middeldorp. "Research Review: Polygenic methods and their application to psychiatric traits." J Child Psychol Psychiatry, 2014, 55(10):1068-1087. doi:10.1111/jcpp.12295

Q. Xiao, Z.J. Liu, S. Tao, Y.-M. Sun, D. Jiang, H.-L. Li, H. Chen, X. Liu, B. Lapin, C.-H. Wang, S.L. Zheng, J. Xu, Z.-Y. Wu. "Risk prediction for sporadic Alzheimer's disease using genetic risk score in the Han Chinese population." Oncotarget. 2015, 6(35):36955-36964. doi:10.18632/oncotarget.6271

C. Xue, H. Sun, G. Hu, W. Qi, Y. Yue, J. Rao, W. Yang, C. Xiao, J. Chen \& the Alzheimer's Disease Neuroimaging Initiative. "Disrupted patterns of rich-club and diverse-club organizations in subjective cognitive decline and amnestic mild cognitive impairment." Front. Neurosci., 2020, https://doi.org/10.3389/fnins.2020.575652.

J.S. Yokohama, L.W. Bonham, R.L. Sears, E. Klein, A. Karydas, J.H. Kramer, B.L. Miller, G. Coppola. "Decision tree analysis of genetic risk for clinically heterogeneous Alzheimer's disease." BMC Neurol, 2015, 15:47. doi:10.1186/s12883-015-0304-6

J.T. Yu, L. Tan, J. Hardy. Apolipoprotein E in Alzheimer's disease: an update." Annu Rev Neurosci, 2014, 37:79-100. doi:10.1146/annurev-neuro-071013-014300

J.P. Yuan, E. Henje Blom, T. Flynn, Y. Chen, T.C. Ho, C.G. Connolly, R.A. Dumont Walter, T.T. Yang, D. Xu, O. Tymofiyeva. "Test-retest reliability of graph theoretical metrics in adolescent brains." Brain Connectivity, 2018, 9(2) :144-154, doi.org/10.1089/brain.2018.0580

Y. Zhang, N. Schuff, G.-H. Jahng, W. Bayne, S. Mori, L. Schad, S. Mueller, A.-T. Du, J.H. Kramer, K. Yaffe, H. Chui, W.J. Jagust, B.L. Miller, M.W. Weiner. "Diffusion tensor imaging of cingulum fibers in mild cognitive impairment and Alzheimer disease." Neurology, 2007, 68, 13-19. doi: 10.1212/01.wnl.0000250326.77323.01 
S. Zhang, D. Han, X. Tan, J. Feng, Y. Guo, Y. Ding. "Diagnostic accuracy of 18 F-FDG and 11 C-PIBPET for prediction of short-term conversion to Alzheimer's disease in subjects with mild cognitive impairment." Int J Clin Pract., 2012, 66(2):185-198. doi:10.1111/j.17421241.2011.02845.x

Y. Zhou, J.H. Dougherty Jr, K.F. Hubner, B. Bai, R.L. Cannon, R.K. Hutson. "Abnormal connectivity in the posterior cingulate and hippocampus in early Alzheimer's disease and mild cognitive impairment." Alzheimer's and Dementia, 2008, 4(4):265-270. doi.org/10.1016/j.jalz.2008.04.006 